\title{
On a Relative Computability Notion for Real Functions
}

\author{
Dimiter Skordev ${ }^{1} \quad$ Ivan Georgiev ${ }^{2}$ \\ ${ }^{1}$ University of Sofia, Bulgaria \\ ${ }^{2}$ Burgas Prof. Assen Zlatarov University, Bulgaria
}

Computability in Europe 2011

29 June 2011 


\section{Abstract}

- For any class $\mathcal{F}$ of total functions in $\mathbb{N}$, we define what it means for a real function to be conditionally $\mathcal{F}$-computable.

This notion extends the notion of uniform $\mathcal{F}$-computability of real functions introduced in the paper [SkWeGe10].

- If $\mathcal{F}$ consists of recursive functions then the conditionally $\mathcal{F}$-computable real functions are computable in the sense of [Gr55] extended by allowing the used computable functionals to be partial and by considering real functions of any number of variables. This yields certain notions of subrecursive computability for real functions.

- Under certain weak assumptions about $\mathcal{F}$, we show that:

- conditional $\mathcal{F}$-computability is preserved by substitution,

- all conditionally $\mathcal{F}$-computable real functions are locally uniformly $\mathcal{F}$-computable,

- the conditionally $\mathcal{F}$-computable real functions with compact domains are uniformly $\mathcal{F}$-computable.

- All elementary functions of calculus are conditionally $\mathcal{M}^{2}$-computable. 


\section{Computability of Real Functions in the Extended Sense of}

\section{$[$ Gr55]}

As in [SkWeGe 10], a triple $(f, g, h)$ of total one-argument functions in $\mathbb{N}$ will be called to name a real number $\xi$ if

$$
\left|\frac{f(t)-g(t)}{h(t)+1}-\xi\right|<\frac{1}{t+1}
$$

for all $t \in \mathbb{N}(h=\lambda t . t$ is actually used in [Gr55]).

Let $N \in \mathbb{N}$ and $\theta: D \rightarrow \mathbb{R}$, where $D \subseteq \mathbb{R}^{N}$. The function $\theta$ is computable in the extended sense of [Gr55] iff there exist recursive operators $F, G, H$ acting on $3 \mathrm{~N}$-tuples of one-argument functions in $\mathbb{N}$ and such that, whenever $\left(\xi_{1}, \ldots, \xi_{N}\right) \in D$ and $\left(f_{1}, g_{1}, h_{1}\right), \ldots$, $\left(f_{N}, g_{N}, h_{N}\right)$ are triples naming $\xi_{1}, \ldots, \xi_{N}$, respectively, the functions $F(\bar{f}, \bar{g}, \bar{h}), G(\bar{f}, \bar{g}, \bar{h}), H(\bar{f}, \bar{g}, \bar{h})$, where $\bar{f}=f_{1}, \ldots, f_{N}$, $\bar{g}=g_{1}, \ldots, g_{N}$, and $\bar{h}=h_{1}, \ldots, h_{N}$, are total, and the triple of them names $\theta\left(\xi_{1}, \ldots, \xi_{N}\right)$. 


\section{Subrecursive Computability of Functions of Reals}

as a Certain Kind of Relative Computability

As far as we know, the first paper in this direction is [TeZi 10]. For any class $\mathcal{F}$ of total functions in $\mathbb{N}$ which satisfies certain conditions, some functions in $\mathbb{R}$ are said to be in $\mathcal{F}$, and some ones are said to be uniformly $\mathcal{F}$. The definition of these notions requires the existence of functions belonging to $\mathcal{F}$ which appropriately produce arbitrarily close rational approximations of the value of the real function in question making use of sufficiently close rational approximations of the values of the arguments of this function.

Further similar papers are [SkWeGe10] and [Skxx], where the notion of uniform $\mathcal{F}$-computability is studied. Its definition is similar to the characterization of computability in the extended sense of [Gr55] on the previous slide, but uses so-called $\mathcal{F}$-substitutional mappings instead of recursive operators. Under the assumptions from [TeZi 10 ] about $\mathcal{F}$, the uniformly $\mathcal{F}$ functions turn out to be exactly the uniformly $\mathcal{F}$-computable ones with open domains. 


\section{$\mathcal{F}$-Substitutional Mappings}

For any $m \in \mathbb{N}$, we will denote by $\mathbb{T}_{m}$ the set of all $m$-argument total functions in $\mathbb{N}$. Let $\mathcal{F} \subseteq \cup_{m \in \mathbb{N}} \mathbb{T}_{m}$. For any $k, m \in \mathbb{N}$, certain mappings of $\mathbb{T}_{1}^{k}$ into $\mathbb{T}_{m}$ will be called $\mathcal{F}$-substitutional, as follows:

1. For any $m$-argument projection function $h$ in $\mathbb{N}$ the mapping $F$ defined by $F\left(f_{1}, \ldots, f_{k}\right)=h$ is $\mathcal{F}$-substitutional.

2. For any $i \in\{1, \ldots, k\}$, if $F_{0}$ is a $\mathcal{F}$-substitutional mapping of $\mathbb{T}_{1}^{k}$ into $\mathbb{T}_{m}$ then so is the mapping $F$ defined by

$$
F\left(f_{1}, \ldots, f_{k}\right)\left(n_{1}, \ldots, n_{m}\right)=f_{i}\left(F_{0}\left(f_{1}, \ldots, f_{k}\right)\left(n_{1}, \ldots, n_{m}\right)\right) .
$$

3. For any $r \in \mathbb{N}$ and $f \in \mathcal{F} \cap \mathbb{T}_{r}$, if $F_{1}, \ldots, F_{r}$ are $\mathcal{F}$-substitutional mappings of $\mathbb{T}_{1}^{k}$ into $\mathbb{T}_{m}$ then so is the mapping $F$ defined by

$$
\begin{aligned}
& \quad F\left(f_{1}, \ldots, f_{k}\right)\left(n_{1}, \ldots, n_{m}\right)= \\
& f\left(F_{1}\left(f_{1}, \ldots, f_{k}\right)\left(n_{1}, \ldots, n_{m}\right), \ldots, F_{r}\left(f_{1}, \ldots, f_{k}\right)\left(n_{1}, \ldots, n_{m}\right)\right) .
\end{aligned}
$$




\section{Two Statements about $\mathcal{F}$-Substitutional Mappings}

Proposition

Let $F: \mathbb{T}_{1}^{k} \rightarrow \mathbb{T}_{m}$ and $G_{1}, \ldots, G_{m}: \mathbb{T}_{1}^{k} \rightarrow \mathbb{T}$, be $\mathcal{F}$-substitutional.

Then so is the mapping $H: \mathbb{T}_{1}^{k} \rightarrow \mathbb{T}_{\text {l }}$ defined by

$$
H(\bar{f})(\bar{n})=F(\bar{f})\left(G_{1}(\bar{f})(\bar{n}), \ldots, G_{m}(\bar{f})(\bar{n})\right),
$$

where $\bar{f}=f_{1}, \ldots, f_{k}$, and $\bar{n}=n_{1}, \ldots, n_{l}$.

Proposition

Let $F: \mathbb{T}_{1}^{k} \rightarrow \mathbb{T}_{m}$ and $G_{1}, \ldots, G_{k}: \mathbb{T}_{1}^{\prime} \rightarrow \mathbb{T}_{p+1}$ be $\mathcal{F}$-substitutional. Then so is the mapping $H: \mathbb{T}_{1}^{\prime} \rightarrow \mathbb{T}_{p+m}$ defined by the equality

$$
H(\bar{g})(\bar{u}, \bar{n})=F\left(\lambda t . G_{1}(\bar{g})(\bar{u}, t), \ldots, \lambda t \cdot G_{k}(\bar{g})(\bar{u}, t)\right)(\bar{n}),
$$

where $\bar{g}=g_{1}, \ldots, g_{l}, \bar{u}=u_{1}, \ldots, u_{p}$, and $\bar{n}=n_{1}, \ldots, n_{m}$. 


\section{Conditional $\mathcal{F}$-Computability of Real Functions}

Let $N \in \mathbb{N}$ and $\theta: D \rightarrow \mathbb{R}$, where $D \subseteq \mathbb{R}^{N}$. The function $\theta$ will be called conditionally $\mathcal{F}$-computable if there exist $\mathcal{F}$-substitutional mappings $E: \mathbb{T}_{1}^{3 N} \rightarrow \mathbb{T}_{1}$ and $F, G, H: \mathbb{T}_{1}^{3 N} \rightarrow \mathbb{T}_{2}$ such that, whenever $\left(\xi_{1}, \ldots, \xi_{N}\right) \in D$ and $\left(f_{1}, g_{1}, h_{1}\right), \ldots,\left(f_{N}, g_{N}, h_{N}\right)$ are triples from $\mathbb{T}_{1}^{3}$ naming $\xi_{1}, \ldots, \xi_{N}$, respectively, the following holds, where $\bar{f}=f_{1}, \ldots, f_{N}, \bar{g}=g_{1}, \ldots, g_{N}$, and $\bar{h}=h_{1}, \ldots, h_{N}$ :

1. There exists a natural number s such that $E(\bar{f}, \bar{g}, \bar{h})(s)=0$.

2. For any natural number $s$ with $E(\bar{f}, \bar{g}, \bar{h})(s)=0$, the number $\theta\left(\xi_{1}, \ldots, \xi_{N}\right)$ is named by the triple

$$
(\lambda t . F(\bar{f}, \bar{g}, \bar{h})(s, t), \lambda t . G(\bar{f}, \bar{g}, \bar{h})(s, t), \lambda t . H(\bar{f}, \bar{g}, \bar{h})(s, t)) .
$$


The Uniformly $\mathcal{F}$-Computable Real Functions are Conditionally $\mathcal{F}$-Computable

Let $N \in \mathbb{N}$, and let the function $\theta: D \rightarrow \mathbb{R}$, where $D \subseteq \mathbb{R}^{N}$, be uniformly $\mathcal{F}$-computable. Then there exist $\mathcal{F}$-substitutional mappings $F^{\circ}, G^{\circ}, H^{\circ}: \mathbb{T}_{1}^{3 N} \rightarrow \mathbb{T}_{1}$ such that, whenever $\left(\xi_{1}, \ldots, \xi_{N}\right) \in D$ and $\left(f_{1}, g_{1}, h_{1}\right), \ldots,\left(f_{N}, g_{N}, h_{N}\right)$ are triples from $\mathbb{T}_{1}^{3}$ naming $\xi_{1}, \ldots, \xi_{N}$, respectively, the number $\theta\left(\xi_{1}, \ldots, \xi_{N}\right)$ is named by the triple $\left(F^{\circ}(\bar{f}, \bar{g}, \bar{h}), G^{\circ}(\bar{f}, \bar{g}, \bar{h}), H^{\circ}(\bar{f}, \bar{g}, \bar{h})\right)$, where $\bar{f}=f_{1}, \ldots, f_{N}, \bar{g}=g_{1}, \ldots, g_{N}$, and $\bar{h}=h_{1}, \ldots, h_{N}$.

To show the conditional $\mathcal{F}$-computability of $\theta$, we set

$$
\begin{aligned}
E(\bar{f}, \bar{g}, \bar{h})(s) & =s, \\
F(\bar{f}, \bar{g}, \bar{h})(s, t) & =F^{\circ}(\bar{f}, \bar{g}, \bar{h})(t), \\
G(\bar{f}, \bar{g}, \bar{h})(s, t) & =G^{\circ}(\bar{f}, \bar{g}, \bar{h})(t), \\
H(\bar{f}, \bar{g}, \bar{h})(s, t) & =H^{\circ}(\bar{f}, \bar{g}, \bar{h})(t) .
\end{aligned}
$$


The Function $\lambda \xi .1 / \xi$ is Conditionally $\mathcal{M}^{2}$-Computable

To prove this, we may set

$$
\begin{aligned}
E(f, g, h)(s) & =(2 h(s)+3) \div(s+1)|f(s)-g(s)|, \\
F(f, g, h)(s, t) & =(h(u(s, t))+1) \operatorname{sg}(f(u(s, t)) \div g(u(s, t))), \\
G(f, g, h)(s, t) & =(h(u(s, t))+1) \operatorname{sg}(g(u(s, t)) \div f(u(s, t))), \\
H(f, g, h)(s, t) & =|f(u(s, t))-g(u(s, t))| \div 1,
\end{aligned}
$$

where $u(s, t)=s+(s+1)^{2}(t+1)$. 


\section{The Function $\lambda \xi \cdot \exp (\xi)$ is Conditionally $\mathcal{M}^{2}$-Computable}

It is proved in $[\mathrm{SkWeGe} 10]$ that $\min (\exp (\xi), \eta)$ is a uniformly $\mathcal{M}^{2}$-computable function of $\xi$ and $\eta$. Hence there exist $\mathcal{M}^{2}$-substitutional mappings $F^{\circ}, G^{\circ}, H^{\circ}: \mathbb{T}_{1}^{6} \rightarrow \mathbb{T}_{1}$ such that, whenever $\left(f_{1}, g_{1}, h_{1}\right)$ and $\left(f_{2}, g_{2}, h_{2}\right)$ are triples from $\mathbb{T}_{1}^{3}$ naming the real numbers $\xi$ and $\eta$, respectively, then $\min (\exp (\xi), \eta)$ is named by the the triple

$\left(F^{\circ}\left(f_{1}, f_{2}, g_{1}, g_{2}, h_{1}, h_{2}\right), G^{\circ}\left(f_{1}, f_{2}, g_{1}, g_{2}, h_{1}, h_{2}\right), H^{\circ}\left(f_{1}, f_{2}, g_{1}, g_{2}, h_{1}, h_{2}\right)\right)$

To see the conditional $\mathcal{M}^{2}$-computability of $\lambda \xi \exp (\xi)$, we may set

$$
\begin{aligned}
E(f, g, h)(s) & =(f(0)+h(0)+1) \div\left((s+1)_{1}(h(0)+1)+g(0)\right), \\
F(f, g, h)(s, t) & =F^{\circ}(f, \lambda x . s+1, g, \lambda x .0, h, \lambda x .0)(t), \\
G(f, g, h)(s, t) & =G^{\circ}(f, \lambda x . s+1, g, \lambda x .0, h, \lambda x .0)(t), \\
H(f, g, h)(s, t) & =H^{\circ}(f, \lambda x . s+1, g, \lambda x .0, h, \lambda x .0)(t),
\end{aligned}
$$

where $(s+1)_{1}$ is the exponent of the prime number 3 in $s+1$. 
The Partial Recursive Functions in $\mathbb{N}$ Regarded as Functions in $\mathbb{R}$ are Conditionally $\mathcal{M}^{2}$-Computable

Let $\theta$ be an $N$-argument partial recursive function. Then $\theta$ has a representation of the form

$$
\theta\left(x_{1}, \ldots, x_{N}\right)=U\left(\mu y\left[T\left(x_{1}, \ldots, x_{N}, y\right)=0\right]\right),
$$

where $T, U \in \mathcal{M}^{2}$. To show the conditional $\mathcal{M}^{2}$-computability of $\theta$, we may set

$$
\begin{aligned}
E(\bar{f}, \bar{g}, \bar{h})(s) & =T\left(x_{1}, \ldots, x_{N}, s\right)+\max _{y<s} \overline{\operatorname{sg}} T\left(x_{1}, \ldots, x_{N}, y\right), \\
F(\bar{f}, \bar{g}, \bar{h})(s, t) & =U(s), \\
G(\bar{f}, \bar{g}, \bar{h})(s, t) & =0, \\
H(\bar{f}, \bar{g}, \bar{h})(s, t) & =0,
\end{aligned}
$$

where $\bar{f}=f_{1}, \ldots, f_{N}, \bar{g}=g_{1}, \ldots, g_{N}, \bar{h}=h_{1}, \ldots, h_{N}$, and

$$
x_{i}=\left\lfloor\frac{f_{i}(1)-g_{i}(1)}{h_{i}(1)+1}+\frac{1}{2}\right\rfloor, \quad i=1, \ldots, N .
$$




\section{Substitution in Conditionally $\mathcal{F}$-Computable Real Functions}

\section{Theorem}

Let the class $\mathcal{F}$ contain the addition function and one-argument functions $L$ and $R$ such that $\{(L(s), R(s)) \mid s \in \mathbb{N}\}=\mathbb{N}^{2}$. Then the substitution operation on real functions preserves conditional $\mathcal{F}$-computability.

As an application, we will show that the function $\theta(\xi)=\ln \xi$ is conditionally $\mathcal{M}^{2}$-computable. Let us consider the function $\theta^{\circ}$ having domain $\left\{\left(\xi_{1}, \xi_{2}\right) \in \mathbb{R}^{2} \mid \xi_{1}>0, \xi_{1} \xi_{2} \geq 1\right\}$ and defined by $\theta^{\circ}\left(\xi_{1}, \xi_{2}\right)=\ln \xi_{1}$. This function is uniformly $\mathcal{M}^{2}$-computable by [SkWeGe 10], hence it is conditionally $\mathcal{M}^{2}$-computable. On the other hand, $\theta(\xi)=\theta^{\circ}(\xi, 1 / \xi)$ for all $\xi \in \operatorname{dom}(\theta)$.

Since the arctan, arcsin, arccos, sine and cosine functions are shown in $[\mathrm{SkWeGe} 10]$ to be uniformly $\mathcal{M}^{2}$-computable, and so are the sum, difference and product functions, as well as the functions $\sqrt[n]{\xi}, n=2,3, \ldots$, we may conclude that all elementary functions of calculus are conditionally $\mathcal{M}^{2}$-computable. 


\section{Local uniform $\mathcal{F}$-computability of the conditionally}

$\mathcal{F}$-computable functions

Let $N \in \mathbb{N}$ and $\theta: D \rightarrow \mathbb{R}$, where $D \subseteq \mathbb{R}^{N}$. The function $\theta$ will be called locally uniformly $\mathcal{F}$-computable if any point of $D$ has some neighbourhood $U$ such that the restriction of $\theta$ to $D \cap U$ is uniformly $\mathcal{F}$-computable.

\section{Theorem}

Let for any $a, b \in \mathbb{N}$ the class $\mathcal{F}$ contain the two-argument function whose value at $(x, y)$ is $b$ or $y$ depending on whether or not $x=a$. Let also all one-argument constant functions in $\mathbb{N}$ belong to $\mathcal{F}$. Then all conditionally $\mathcal{F}$-computable real functions are locally uniformly $\mathcal{F}$-computable.

This theorem and a characterization theorem from $[S k x x]$ imply that, under the assumptions about $\mathcal{F}$ in them, if $\theta$ is a conditionally $\mathcal{F}$-computable function then each point of $\operatorname{dom}(\theta)$ has some neighbourhood $U$ such that $\theta$ is uniformly continuous in $\operatorname{dom}(\theta) \cap U$. 


\section{Some Computable Real Functions which are not}

Conditionally $\mathcal{F}$-Computable, whatever be the Class $\mathcal{F}$

Let $\theta: \mathbb{R} \backslash\left\{1, \frac{1}{2}, \frac{1}{3}, \ldots\right\} \rightarrow \mathbb{R}$ be defined by

$$
\theta(\xi)=\sum_{k=1}^{\infty} \frac{1}{2^{k}} \sigma\left(\xi-\frac{1}{k}\right),
$$

where $\sigma$ is the restriction of the sign function to $\mathbb{R} \backslash\{0\}$. The function $\theta$ is computable in the extended sense of [Gr55], but there exists no neibourhood $U$ of 0 such that $\theta$ is uniformly continuous in $\operatorname{dom}(\theta) \cap U$. By the statement in the last paragraph of the previous slide, $\theta$ is not conditionally $\mathcal{F}$-computable for $\mathcal{F}=\cup_{m \in \mathbb{N}} \mathbb{T}_{m}$, therefore it is not conditionally $\mathcal{F}$-computable, whatever be the class $\mathcal{F}$ of total functions in $\mathbb{N}$.

Another similar function is the one obtained from the elementary function $\xi \arctan \left(\tan \frac{1}{\xi}\right)$ by extending it as 0 for $\xi=0$. 


\section{Uniform $\mathcal{F}$-computability of the locally uniformly $\mathcal{F}$-computable functions with compact domains}

Theorem

Let the class $\mathcal{F}$ be closed under substitution, and let $\mathcal{F}$ contain the projection functions, the successor function, the addition function, the function $\lambda x y \cdot x \dot{-} y$ and the function $\lambda x y \cdot x(1 \dot{-y})$. Then all locally uniformly $\mathcal{F}$-computable real functions with compact domains are uniformly $\mathcal{F}$-computable.

Corollary

Under the assumptions of the above theorem, all conditionally $\mathcal{F}$-computable real functions with compact domains are uniformly $\mathcal{F}$-computable. 


\section{Some Comments}

The conditional $\mathcal{F}$-computability of real functions has some similarity in its spirit with the notion of a real function in $\mathcal{F}$ introduced in [TeZi 10]. However, there are essential differences between the two notions. For instance, if $\mathcal{F}$ is the class of the lower elementary functions then:

- the class of the real functions in $\mathcal{F}$ is not closed under substitution;

- there are elementary functions of calculus which are not in $\mathcal{F}$;

- there are real functions in $\mathcal{F}$ which are not computable in the extended sense of [Gr55]. 


\section{References}

[Gr55] Grzegorczyk, A.: Computable functionals. Fund. Math., 42, 168-202 (1955)

园 [SkWeGe 10] Skordev, D., Weiermann, A., Georgiev, I.: $\mathcal{M}^{2}$-computable real numbers. J. Logic Comput. (Advance Access published September 21, 2010), doi:10.1093/logcom/exq050

圆 [Skxx] Skordev, D.: Uniform computability of real functions. In: Collection of Summaries of Talks Delivered at the Scientific Session on the Occasion of the 120th Anniversary of FMI (Sofia, October 24, 2009) (to appear)

[TeZi 10] Tent, K., Ziegler, M.: Computable functions of reals. Münster J. Math., 3, 43-66 (2010) (An earlier version appeared at arxive.org in March 2009 under the title "Low functions of reals") 